\title{
Increased Th17 cells and IL-17A
}

(a) CrossMark exist in patients with $B$ cell acute lymphoblastic leukemia and promote proliferation and resistance to daunorubicin through activation of Akt signaling

\author{
Laixi $\mathrm{Bi}^{1+}$, Junqing $\mathrm{Wu}^{1+}$, Aifang $\mathrm{Ye}^{2}$, Jianbo $\mathrm{Wu}^{2}$, Kang $\mathrm{Yu}^{1}$, Shenghui Zhang ${ }^{1 *}$ and Yixiang $\mathrm{Han}^{2 *}$
}

\begin{abstract}
Background: Immune regulation is crucial for the pathogenesis of B-cell acute lymphoblastic leukemia (B-ALL). It has been reported that Th17 cells as a newly identified subset of $C D 4^{+} T$ cells are involved in the pathogenesis of several hematological disorders. However, the role of Th17 cells in the pathophysiology of B-ALL is still unclear.

Methods: The frequencies of T cells were determined by flow cytometry in the peripheral blood and bone marrow of 44 newly diagnosed B-ALL patients and 25 age-matched healthy donors. The cell viability and apoptosis were determined by CCK-8 assay and Annexin $V$ staining, respectively. Western blot was applied to identify the level of Akt and Stat3 phosphorylation.

Results: We assessed and observed a significantly increased frequency of Th17 cells and a drastically decreased frequency of Th1 cells in peripheral blood mononuclear cells and bone marrow mononuclear cells from newly diagnosed B-ALL patients compared with healthy donors. Furthermore, increased levels of Th17-related cytokines including IL-17, IL-21, IL-23, IL-1 $\beta$, and IL-6 were presented in between blood and marrow in B-ALL patients. Both IL-17A and IL-21, two Th17-secreted cytokines, induced the proliferation of B-ALL cell line Nalm- 6 and patient B-ALL cells isolated from B-ALL patients, herein either cytokine led to the phosphorylation of Akt and Stat3. Additionally, IL-17A promoted resistance to daunorubicin via activation of Akt signaling and the PI3K/Akt inhibitor LY294002 or perifosine almost completely rescued daunorubicin-induced cell death in B-ALL cells.

Conclusions: Our findings suggest that elevated Th17 cells secrete IL-17A by which promotes the proliferation and resistance to daunorubicin in B-ALL cells through activation of Akt signaling. Th17 cells may represent a novel target to improve B-ALL immunotherapy.
\end{abstract}

Keywords: B-cell acute lymphoblastic leukemia, Th17 cells, IL-17A, Th1 cells

\footnotetext{
*Correspondence: shenghuizhang1@126.com;

yxhan2001@163.com

${ }^{\dagger}$ Laixi Bi and Junqing Wu contributed equally to this work

${ }^{1}$ Department of Hematology, The First Affiliated Hospital of Wenzhou Medical University, Wenzhou 325015, China

${ }^{2}$ Laboratory of Internal Medicine, the First Affiliated Hospital of Wenzhou Medical University, Wenzhou 325015, China

Full list of author information is available at the end of the article
} 


\section{Background}

B-cell acute lymphoblastic leukemia (B-ALL) characterized by accumulation of immature lymphoid progenitors affects both children and adults. Great strides in development of effective treatments have led to a cure rate of more than $90 \%$ in children, whereas the prognosis of adults remains poor [1]. Relapses, treatment resistance and serious infections are the leading causes of death in most cases. Pro-survival signals provided by tissue microenvironments, such as intricate crosstalk between B-ALL cells and $\mathrm{CD} 4^{+} \mathrm{T}$ cells, mesenchymal stromal cells, and various cytokines, contribute to maintain leukemic clones and promote resistance to chemotherapy in adult B-ALL patients [2].

Undoubtedly, immune dysfunction occurs in the bone marrow (BM) microenvironment of patients with B-ALL due to abnormal infiltrations of several immune cells. As one of the most important immune cells, $\mathrm{CD}^{+}{ }^{+} \mathrm{T}$ cells are vital in the induction and control the differentiation of other immune cells in response to tumor-specific antigens and play a central role in initiating, regulating, and maintaining immune responses against cancer. Th1 cells produce interferon- $\gamma$ (IFN- $\gamma$ ) and predominantly promote cell-mediated immunity, whereas Th2 cells secrete IL-4, IL-5 and IL-13. Th17 cells, named for their signature production of IL-17A, also secrete IL-17F, IL-21, and IL-22, thereby inducing an enormous tissue reaction due to the broad distribution of the IL-17 and IL-21 receptors and exerting a crucial role in the development of inflammatory and autoimmune diseases [3-6]. In addition, Th17 cells are implicated in many cancers, but their role in cancer has not been well elucidated and remains under debate [7-10]. It is now well accepted that Th1 cells and their secreted signature cytokine IFN- $\gamma$ exhibit robust anti-tumor activity $[11,12]$. Several studies have shown that IFN- $\gamma$ inhibits Th17 cell differentiation and IL-17 inhibits Th1 cell differentiation and IFN- $\gamma$ production [7, 13-15], suggesting that Th1 and Th17 cells appear to have a reciprocal relation in function. Therefore, it makes sense to investigate the interplay of Th1 and Th17 cells in the tumor microenvironment. B-ALL represents an ideal model to assess the crosstalk between tumor and the immune system as the disease is usually widely disseminated, so that immune cells in the peripheral blood (PB) and BM are in close contact with B-ALL cells. The immune system fails to identify and eliminate B-ALL cells, by which contributes to the development of B-ALL.

In the present study, we demonstrate that elevated frequencies of Th17 cells and Th17-related cytokines and reduced frequencies of Th1 cells are presented in $\mathrm{PB}$ and BM from B-ALL patients; Th17-secreted cytokines IL-17A and IL-21 activate the Akt signaling and Stat3 signaling, and subsequently stimulate the proliferation in
B-ALL cell line Nalm-6 and primary B-ALL cells; IL-17A also promotes resistance to daunorubicin through activation of Akt signaling.

\section{Methods \\ Patients and samples}

A total of 44 patients with newly diagnosed B-ALL (age range 15-68 years, median age 33 years) were enrolled in this study from July 2009 and February 2015 in our center. All patients with B-ALL were diagnosed according to the World Health Organization classification system [16]. Twenty-five age-matched healthy donors for control were simultaneously enrolled in this study. This study was approved by the Institutional Review board of the First Affiliated Hospital of Wenzhou Medical University and informed consent was obtained from all participants in accordance with the Declaration of Helsinki protocol.

\section{Flow cytometric analysis}

The frequencies of Th17 cells and Th1 cells were analyzed as previously described [7]. Briefly, peripheral blood mononuclear cells (PBMCs) and bone marrow mononuclear cells (BMMCs) from B-ALL patients and healthy donors were stimulated with $40 \mathrm{ng} / \mathrm{ml}$ phorbol 12-myristate13-acetate (PMA) and $1 \mu \mathrm{g} / \mathrm{ml}$ ionomycin for $5 \mathrm{~h}$ in the presence of $1 \mu \mathrm{g} / \mathrm{ml}$ brefeldin A (BFA; all from Sigma-Aldrich, St.louis, MO, USA). The cells were subsequently surface stained with a combination of PerCP-conjugated anti-CD3 and FITC-conjugated antiCD8, fixed and permeabilized and intracellularly stained with PE-conjugated anti-IL-17A and APC-conjugated anti-IFN- $\gamma$ (all from BD Biosciences, San Jose, CA, USA). Stained cells were acquired and analyzed using CellQuest software on a FACSCalibur instrument (BD Biosciences).

\section{ELISA for cytokine measurements}

The plasma samples were obtained from $\mathrm{PB}$ and BM after centrifugation and stored at $-80{ }^{\circ} \mathrm{C}$ until used. Human IL-17 and IL-21 were assayed using ELISA kits purchased from BioLegend (San Diego, CA, USA) with the assay sensitivities of 2.0 and $16.0 \mathrm{pg} / \mathrm{ml}$, respectively. Human IL-23, IL-1 $\beta$, and IL- 6 were measured using ELISA kits purchased from R\&D systems (Minneapolis, MN, USA) with the assay sensitivities of $15.0,1.0$, and $0.7 \mathrm{pg} / \mathrm{ml}$, respectively.

\section{Quantitative RT-PCR for gene expression analysis}

Total RNA was extracted from mononuclear cells using TRIzol reagent (Invitrogen, Carlsbad, CA, USA) and was subsequently reverse transcribed into cDNA according to the manufacturer's guidelines. Then, quantitative PCR was performed using SYBR Green PCR master mix 
(TaKaRa, Dalian, China) with respective primer pairs (for primers sequences, see Additional file 1: Table S1) in triplicate on an ABI 7500 Real-Time instrument. Expression data were normalized to GAPDH as an endogenous control and the relative expression levels were evaluated using the $2^{-\Delta \Delta \mathrm{Ct}}$ method.

\section{Cell Counting Kit-8 (CCK-8) assay and apoptosis assay} $\mathrm{CD} 19^{+}$cells were isolated from PBMCs or BMMCs of B-ALL patients (thereafter as patient B-ALL cells) using B Cell Isolation Kit II (Miltenyi Biotec, Bergisch Gladbach, Germany) according to the manufacture's protocols. B-ALL cell line Nalm-6 was a gift from Dr. Chiqi Chen (School of Medicine, Jiaotong University, Shanghai, China). After Nalm-6 cells and patient B-ALL cells were treated or untreated with IL-17, IL-21 (both from Peprotech, Rocky Hill, NJ, USA), daunorubicin, LY294002 or perifosine (Selleck Chemicals, Houston, TX, USA), the cell viability and apoptosis were measured. The cell viability was determined using CCK- 8 assay (Dojindo, Kumamoto, Japan) according to the manufacturer's instruction. The absorbance was read at $450 \mathrm{~nm}$ using an ELISA reader (ELx800; Bio-Tek Instruments, Winooski, VT, USA). The apoptosis was determined using the Annexin V binding assay as previously described [17].

\section{Western blot assays}

Western blot analysis was performed as previously described $[18,19]$. Briefly, after treatment with IL-17A or IL-21, Nalm-6 cells and patient B-ALL cells were collected, lysed, and further subjected to western blot with antibodies specific for phospho-Akt (Ser473), Akt, GAPDH (Cell Signaling Technology, Beverly, MA, USA), phospho-Stat3 (Tyr705), or Stat3 (Bioworld Technology, St.Louis Park, MN, USA), respectively.

\section{Induction assay}

$\mathrm{CD}^{+} \mathrm{T}$ cells were isolated from PBMCs of B-ALL patients using $\mathrm{CD}^{+} \mathrm{T}$ cell isolation kit (Miltenyi Biotec) according to the manufacturer's instructions. $\mathrm{CD} 4^{+} \mathrm{T}$ cells $\left(2 \times 10^{5}\right)$ were cultured in $1 \mathrm{ml}$ of complete medium in 48-well plates stimulated with plate-bound anti-CD3 antibody (OKT3, $4 \mu \mathrm{g} / \mathrm{ml}$; eBioscience, San Diego, CA,
USA) and IL-2 (300 units/ml) with or without Nalm-6 cells. After 14 days, Th17 cells were determined after stimulation with PMA and ionomycin in the presence of BFA.

\section{Statistical analysis}

Data are presented as mean \pm SEM, and statistical analyses were performed by a one-way analysis of variance or Mann-Whitney test. $P$ values less than 0.05 were considered statistically significant.

\section{Results \\ Increased Th17 cells and decreased Th 1 cells in B-ALL patients}

Th17 cells have been reported to be enriched in hematological malignancies including acute myeloid leukemia, multiple myeloma, and T-cell acute lymphoblastic leukemia $[7,15,20,21]$. To investigate whether Th17 cells are also enriched in B-ALL, we evaluated the frequency of Th17 cells based on cytokine patterns after in vitro stimulation with PMA plus ionomycin in short-term culture. As shown in Fig. 1a, b, the frequencies of Th17 cells were $3.5 \pm 0.46 \%$ in B-ALL PBMCs compared with $1.8 \pm 0.21 \%$ in healthy donor PBMCs $(P<0.01)$, and $3.2 \pm 0.32 \%$ in B-ALL BMMCs compared with $1.4 \pm 0.26 \%$ in healthy donor BMMCs $(P<0.01)$, suggesting that Th17 cells are highly enriched in both $\mathrm{PB}$ and BM from B-ALL patients. We simultaneously analyzed the frequency of Th1 cells and found that the frequency of Th1 cells was significantly decreased in both PBMCs and BMMCs from B-ALL patients compared with those from healthy donors (Fig. 1a and b). Quantitative RT-PCR was used to measure the mRNA levels of IL-17A and IFN- $\gamma$ in both PBMCs and BMMCs and found that increased expression of IL-17A and decreased expression of IFN- $\gamma$ were presented in both PBMCs and BMMCs from B-ALL patients compared with those from healthy donors (Fig. 1c). Taken together, these findings suggest that Th17 cells are increased and Th1 cells are decreased in both $P B$ and BM from B-ALL patients. Furthermore, when B-ALL patients achieved complete remission, the frequencies of Th17 cells were markedly decreased in BM compared with those from previously untreated patients (Fig. 1d).

\footnotetext{
(See figure on next page.)

Fig. 1 Th17 cells increase with reduced Th1 cells in freshly isolated PBMCs and BMMCs from patients with B-ALL. a PBMCs and BMMCs were separated and stimulated with PMA and ionomycin for $5 \mathrm{~h}$ in the presence of brefeldin A and subsequently stained with antibodies against CD3, CD8, intracellular IL-17A or IFN- $\gamma$. Flow cytometric analysis was used to determine the Th17 and Th1 cell frequencies. Representative dot plots using matching peripheral blood and bone marrow samples from B-ALL patients and healthy donors (HD) were shown. b Statistical data for frequencies of Th17 and Th1 cells within CD4 ${ }^{+} \mathrm{T}$ population were shown. c Total RNA was extracted from CD4 ${ }^{+} \mathrm{T}$ cells isolated from B-ALL patients and HDs and reverse transcribed into CDNA and subsequently determined for IL-17A and IFN- $\gamma$ mRNA expression using quantitative PCR. $\mathbf{d}$ The frequencies of Th17 cells were significantly decreased in BM when B-ALL patients achieved complete remission (CR). e CD4 ${ }^{+} \mathrm{T}$ cells were cultured with or without Nalm- 6 cells for 14 days in the presence of OKT3 plus IL-2 (300 units/ml). Then, frequencies of Th17 cells were determined after stimulation with PMA plus ionomycin
} 

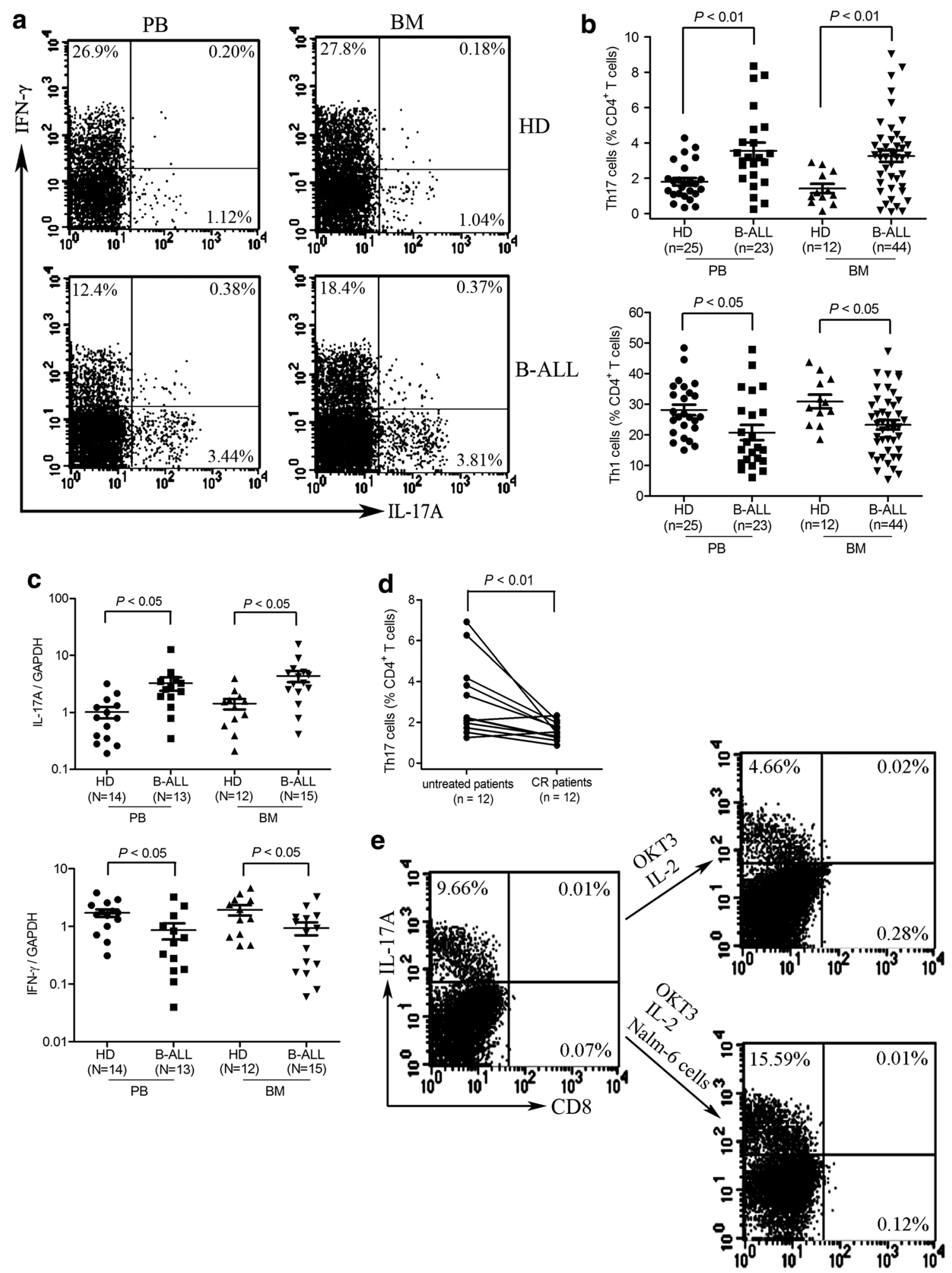
Because increased Th17 cells were presented in B-ALL patients, we next investigated whether B-ALL cells drive the expansion of Th17 cells. We cultured bulk $\mathrm{CD}^{+} \mathrm{T}$ cells from B-ALL patients in the presence of IL-2 in OKT3-coated plates with or without Nalm-6 cells. As shown in Fig. 1e, the percentage of Th17 cells increased in $\mathrm{CD} 4^{+} \mathrm{T}$ cells cultured with Nalm- 6 cells in the presence of OKT3 plus IL-2, whereas the percentage of Th17 cells decreased in $\mathrm{CD}^{+}{ }^{+} \mathrm{T}$ cells cultured with OKT3 plus IL-2. These data indicate that the expansion of Th17 cells may be attributed to the interplay with B-ALL cells.

\section{Th17 cell-related cytokines in B-ALL patients}

IL-17A is the signature cytokine secreted by Th17 cells and contributes to Th17-mediated diseases. IL- 21 is produced by Th17 cells and promotes or sustains Th17 lineage commitment [22]. IL-23, IL-1 $\beta$, and IL-6 regulate the establishment and clonal expansion of Th17 cells. To further confirm elevated existence of Th17 cells in B-ALL patients, we measured the levels of Th17-related cytokines. We observed significant increases in levels of plasma IL-17A and IL-21 in PB and BM from newly diagnosed B-ALL patients compared with those from healthy donors (Fig. 2a and b). Higher levels of IL-23, IL- $1 \beta$, and IL- 6 were also observed in PB and BM from B-ALL patients compared with those from healthy donors (Fig. 2c-e). Taken together, these findings suggest that elevated Th17 cells appear to exist in the PB and BM microenvironment in B-ALL patients.

Two Th17-related cytokines, IL-17A and IL-21, promote the proliferation of B-ALL cells via activation of the Akt and Stat3 signaling

As Th17 cells secrete a variety of cytokines, including IL-17A, IL-17F, IL-21, and IL-22, their effects on B-ALL
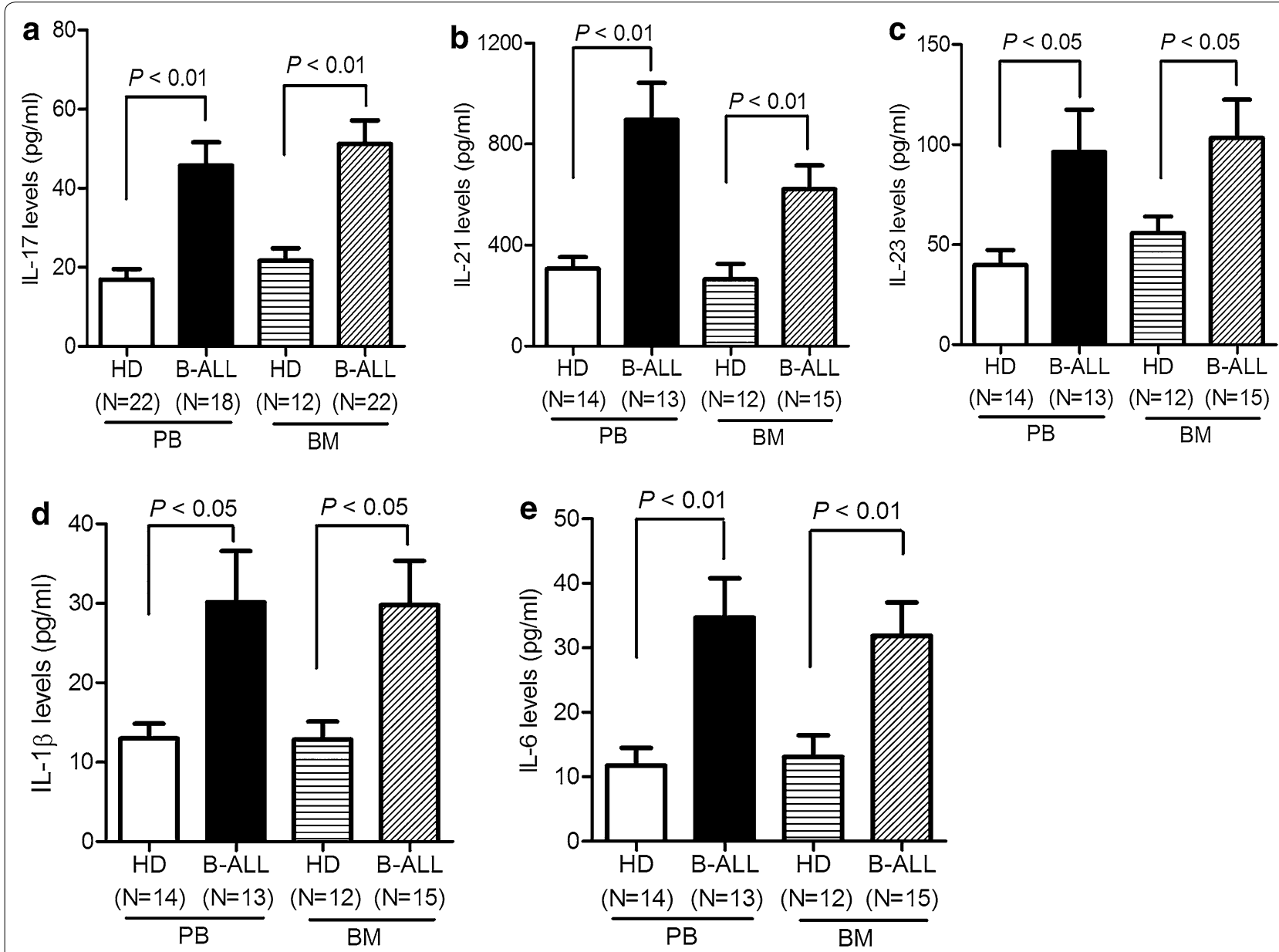

Fig. 2 The levels of Th17-associated cytokines were increased in PB and BM samples from patients with B-ALL. The PB and BM samples were aspirated from B-ALL patients and healthy donors (HD) and determined for the levels of IL-17 (a), IL-21 (b), IL-23 (c) , IL-1 $\beta$ (d), and IL-6 (e) using ELISA. Statistical data were expressed as mean \pm SEM 
cells could be attributed to the actions of any one or a combination of these cytokines. We have demonstrated that B-ALL cell line Nalm-6 promotes the differentiation and expansion of Th17 cells (Fig. 1e), which evokes our interest to investigate whether Th17 cells have a mutual effect on B-ALL cells. As shown in Fig. 3a and b, IL-17A potently promoted the proliferation of patient B-ALL cells and Nalm- 6 cells. IL-21, another main cytokine secreted by Th17 cells, also promoted the proliferation of patient B-ALL cells and Nalm-6 cells (Fig. 3c and d). It has been reported that interleukins exert their effects via specific cell surface receptors. Therefore, we measured the expression of IL-17 receptor A (IL-17RA) and found that the IL-17RA was weakly expressed on patient B-ALL cells and Nalm- 6 cells (Fig. 3e). We used anti-IL-17R antibody to further determine the effect of IL-17RA signaling in IL-17-induced B-ALL cell proliferation. As shown in Fig. 3f, although anti-IL-17R antibody alone did not significantly affect cell viability, it significantly inhibited the proliferation of B-ALL cells in the presence of IL-17A.

Both the PI3K/Akt and Jak2/Stat3 signaling have been shown to play pivotal roles in tumor cell proliferation, survival, invasion and immunosuppression in many tumors including leukemia [23, 24]. Therefore, we analyzed these two signalings in B-ALL cells stimulated with IL-17A or IL-21. As shown in Fig. 3g and h, IL-17A or IL-21 stimulation promoted the phosphorylation of Akt and Stat 3 in Nalm- 6 cells. These findings indicate that Th17 cells exhibit a pro-tumor effect on B-ALL cells through secreting IL-17 and IL-21.

\section{IL-17A promotes resistance to daunorubicin via activation of the Akt signaling}

We had demonstrated above that IL-17A promoted the proliferation of B-ALL cells through activation of the Akt signaling (Fig. 3). As the PI3K/Akt signaling is implicated in resistance to anthracycline-based therapies [25], we tested whether IL-17A mediates resistance to daunorubicin, a chemotherapeutic drug for the treatment of B-ALL. Thus, Nalm- 6 cells and patient B-ALL cells were pre-incubated with IL-17A and subsequently treated with daunorubicin. As shown in Fig. 4a and b, IL-17A treatment attenuated daunorubicin-induced cytotoxicities in Nalm- 6 cells and patient B-ALL cells. Specifically, IL-17A also suppressed daunorubicin-induced apoptosis (Fig. 4c, d; Additional file 2: Figure S1, Additional file 3: Figure S2). To delineate the role of PI3K/Akt signaling in IL-17A-mediated resistance to daunorubicin, we treated these cells with the PI3K/ Akt inhibitor LY294002 or perifosine. LY294002 or perifosine could abrogate IL-17A-mediated protection from daunorubicin-induced cell death in Nalm- 6 cells whereas these two PI3K/Akt inhibitors alone almost did not affect cytotoxicity (Fig. 5a, b). LY294002 or perifosine also abrogated resistance to daunorubicin in patient B-ALL cells induced by IL-17A (Fig. 5c, d). Taken together, these data suggest that IL-17A protects from daunorubicin-induced cell death through activation of the Akt signaling.

\section{Discussion}

Th17 cells contribute to protection against fungal and parasitic infections and participate in various inflammatory and autoimmune diseases. Furthermore, Targeted IL-17 depletion has already been accepted as a therapeutic strategy and is now being tested in clinical trials for several human autoimmune diseases $[26,27]$. Whether Th17 cells and IL-17 also play similar roles in tumor pathogenesis and progression deserves further study. Here we demonstrate that increased Th17 cells and decreased Th1 cells are presented in PB and BM from B-ALL patients. IL-17A or IL-21, two cytokines mainly produced by Th17 cells, can promote the proliferation of B-ALL cells.

The interplay between immune cells and tumor cells contributes importantly in the induction of immune tolerance and tumor progression at tumor sites. Elevated proportions of Th17 cells have been identified in the total $\mathrm{CD}^{+} \mathrm{T}$ cell populations in several different human solid tumors and hematological malignancies including ovarian, prostate carcinomas, multiple myeloma, and acute myeloid leukemia [7, 20, 28, 29]. Abousamra NK et al. [30] have reported that Th17 cells were increased in PB in newly diagnosed ALL patients and a significantly longer overall survival in patients with high Th17 levels. Similarly, the present study also demonstrated that Th17 cells were increased in both $\mathrm{PB}$ and $\mathrm{BM}$ in newly diagnosed patients with B-ALL, a main subtype of ALL [1], indicating that B-ALL cells secrete critical cytokines required for the differentiation and expansion of Th17 cells. Some cytokines, such as IL-1 $\beta$, IL- 6 , and IL-23, have been shown to promote the generation and differentiation of human Th17 cells [7]. Higher levels of IL-23, IL-1 $\beta$, and IL- 6 were observed in B-ALL patients, suggesting that the milieu contributes to the expansion of Th17 cells.

Malignant B cells isolated from B-cell NHL patients have been shown to induce the differentiation of regulatory $\mathrm{T}$ cells in vitro [31]. In the present study, B-ALL cell line Nalm- 6 also promoted the differentiation and expansion of Th17 cells from $\mathrm{CD}^{+} \mathrm{T}$ cells, similar to ovarian tumor cells [28]. Undoubtedly, Th17 cells also secrete cytokines to affect B-ALL cells. It has been well identified that IL-17A plays an important role in promoting cancer growth. The PI3K/Akt and Jak2/Stat3 signaling are two important signalings implicated in IL-17A-mediated proliferation. In the present study, IL-17A stimulation 

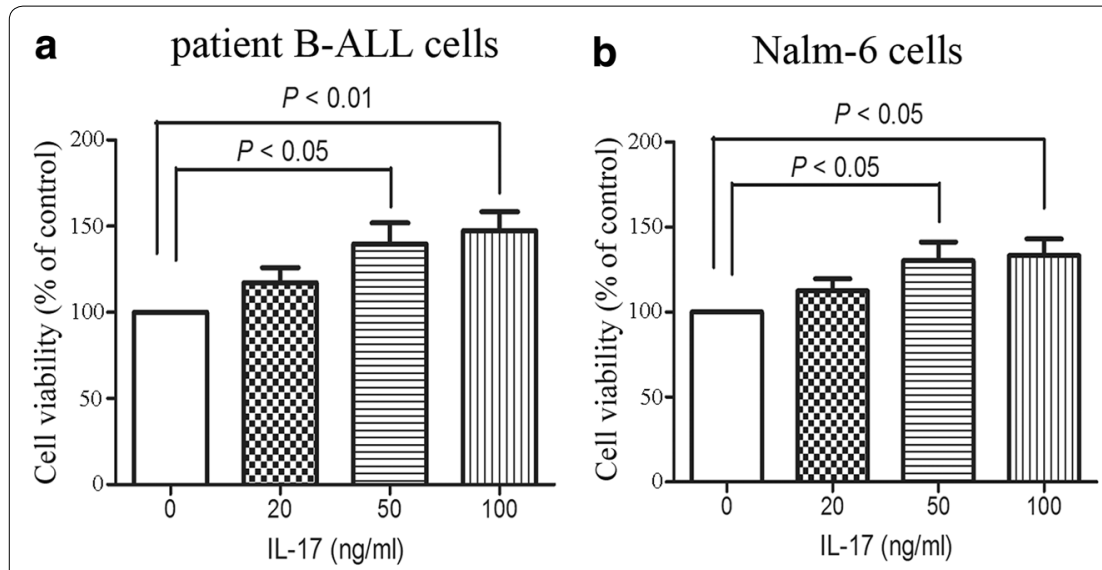

c patient B-ALL cells

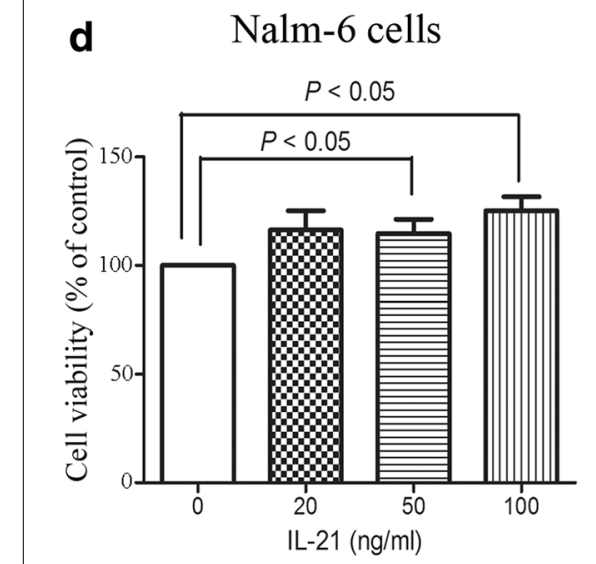

e patient B-ALL cells
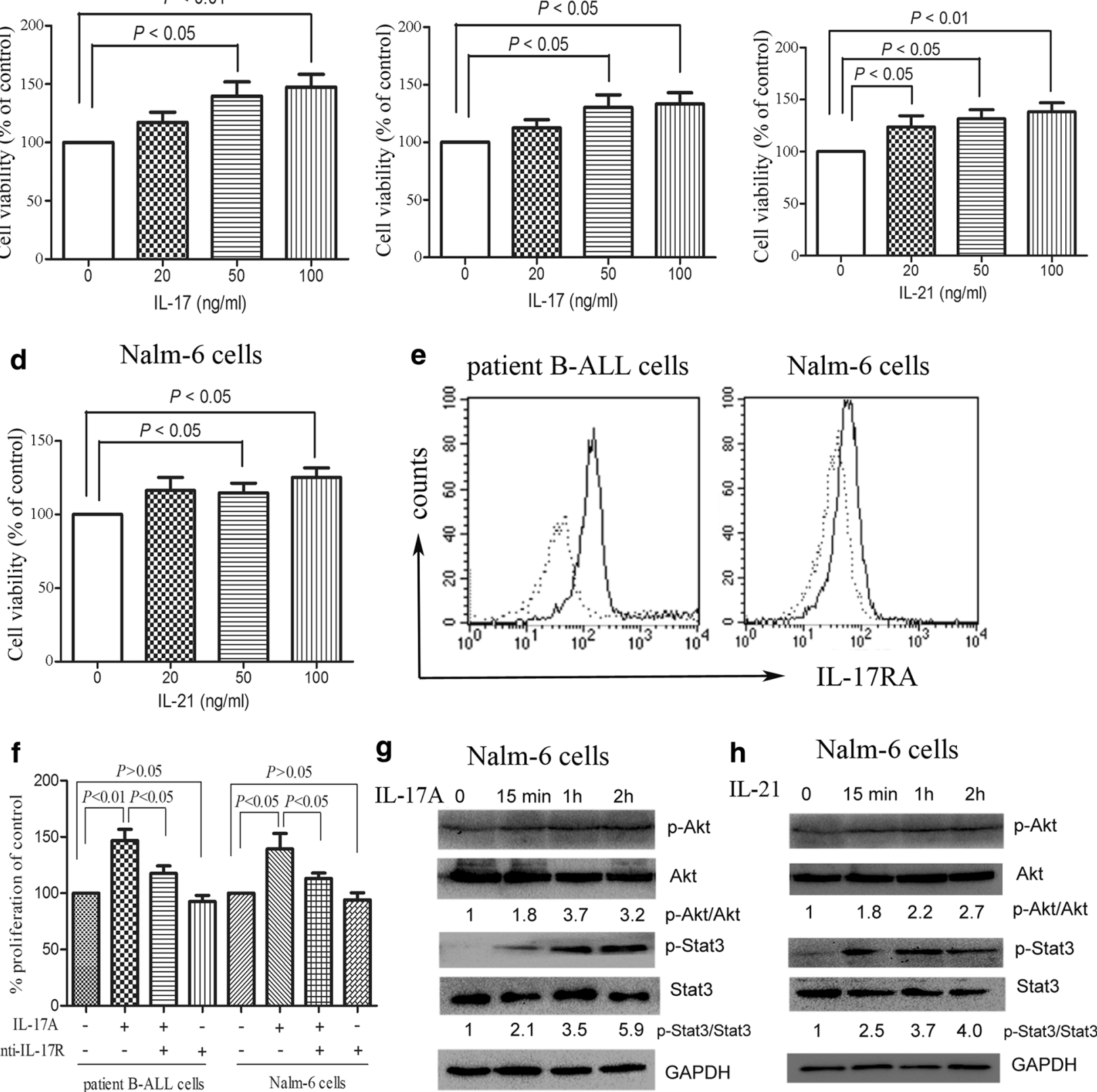

g Nalm-6 cells

IL-17A $0 \quad 15 \min 1 \mathrm{~h} \quad 2 \mathrm{~h}$

h Nalm-6 cells

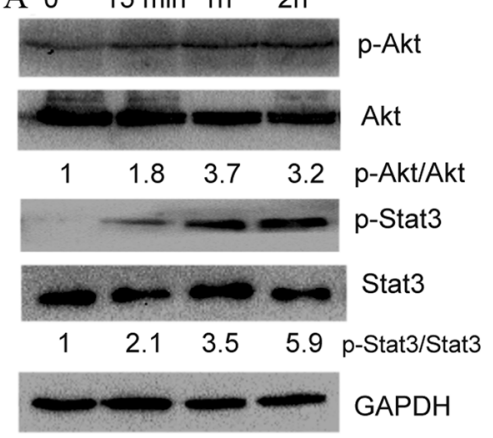

\section{$\mathrm{IL}-21 \quad 0 \quad 15 \mathrm{~min} 1 \mathrm{~h} \quad 2 \mathrm{~h}$}

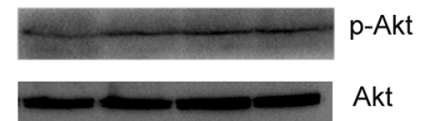

$\begin{array}{lllll}1 & 1.8 & 2.2 & 2.7 & \mathrm{p}-\mathrm{Akt} / \mathrm{Akt}\end{array}$

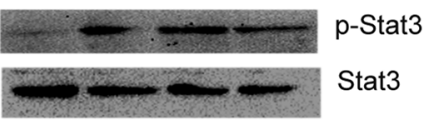

$\begin{array}{lllll}1 & 2.5 & 3.7 & 4.0 & \mathrm{p}-\text { Stat3/Stat3 }\end{array}$

$-\square$ GAPDH

Fig. 3 IL-17A promotes the proliferation of B-ALL cells via activation of IL-17R signaling. a-d Patient B-ALL cells isolated from 4 B-ALL patients and B-ALL cell line Nalm- 6 were incubated in the presence or absence of IL-17A (50 ng/ml) or IL-21 (50 ng/ml) for 7 days and then, CCK-8 assay was used to assess cell viability. Statistical data representing at least three independent experiments are presented as the viability in the presence of IL-17A or IL-21 compared with control. e Representative histograms were presented for the IL-17RA expression of patient B-ALL cells and Nalm-6 cells, determined using PE-conjugated anti-CD217 antibody (solid line) or mouse lgG1 antibody (dotted line) by flow cytometry. f Patient B-ALL cells and Nalm-6 cells were incubated with or without IL-17A $(50 \mathrm{ng} / \mathrm{ml})$ in the presence or absence of anti-IL-17R antibody $(3 \mu \mathrm{g} / \mathrm{ml})$ for 7 days and then, the cell viability was determined using CCK-8 assay. $\mathbf{g}, \mathbf{h}$ Western blot analysis showed the phosphorylation of Akt and Stat3 were prominently increased within $2 \mathrm{~h}$ in Nalm-6 cells stimulated with IL-17A (50 ng/ml) or IL-21 (50 ng/ml). Images representing four independent experiments were shown 

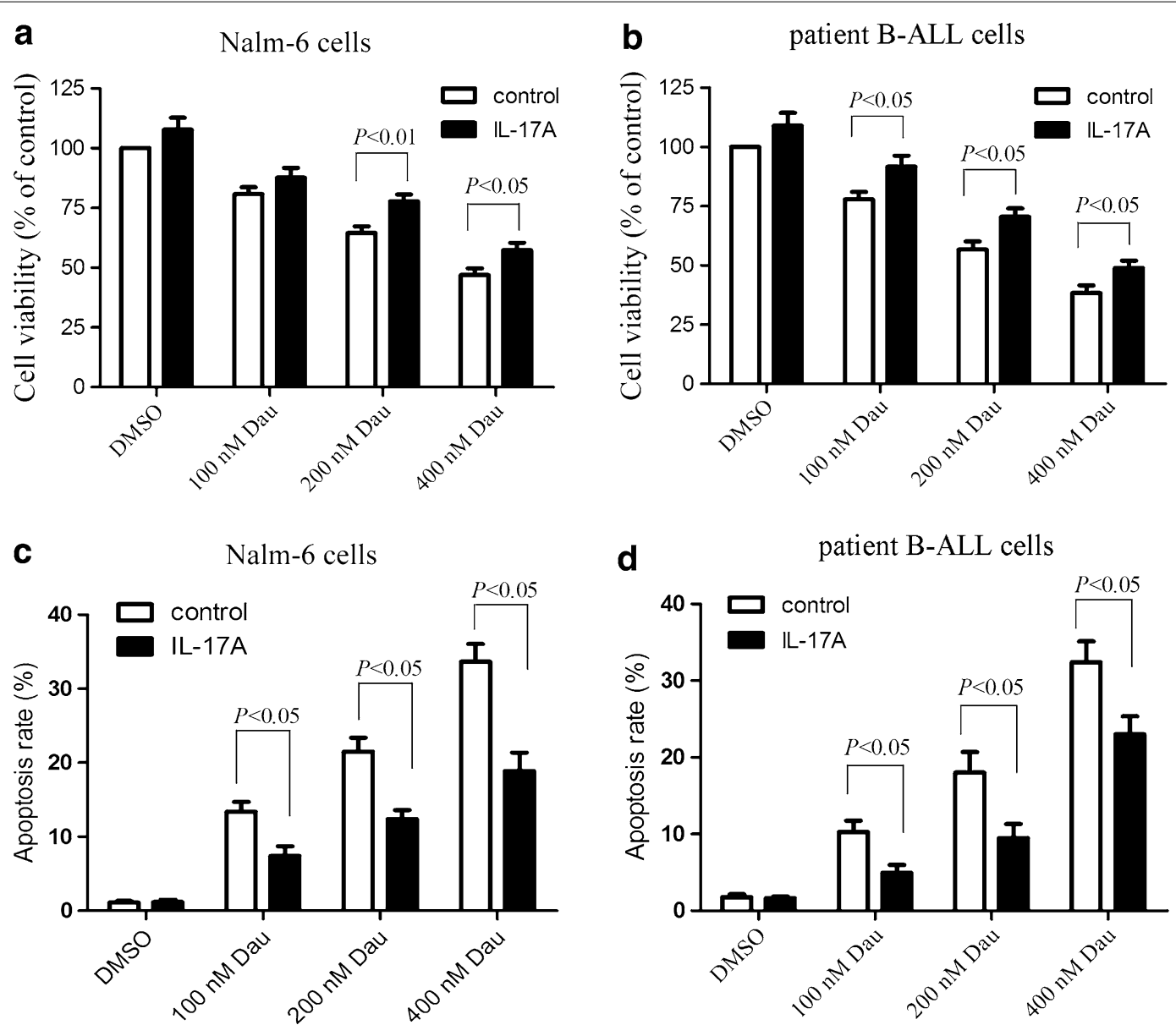

Fig. 4 IL-17A promotes resistance to daunorubicin. Nalm- 6 cells and patient B-ALL cells isolated from 4 B-ALL patients were cultured for $24 \mathrm{~h}$ in complete medium with or without IL-17A at $50 \mathrm{ng} / \mathrm{ml}$ and subsequently supplemented with daunorubicin at 100, 200, or $400 \mathrm{nM}$ as indicated for $24 \mathrm{~h}$. The cell viability was determined using CCK-8 assay, and apoptosis was determined using Annexin $\mathrm{V}$ staining. IL-17A promoted the proliferation and reduced apoptosis of Nalm-6 cells $(\mathbf{a}, \mathbf{c})$ and patient B-ALL cells $(\mathbf{b}, \mathbf{d})$. Statistical data representing at least 3 independent experiments were shown

promoted the proliferation of B-ALL cells through activation of Akt and Stat3 signaling. Obviously, blockade of IL-17RA signaling almost completely abrogated IL17A-induced proliferation in B-ALL cells.

In healthy B cells, IL-21 induces differentiation of naïve and memory $\mathrm{B}$ cells into antibody-producing plasma cells [32]. However, IL-21 exerts diverse effects based on the histology of malignant B cells. For example, IL-21 stimulation induces apoptosis of chronic lymphocytic leukemia and diffuse large B-cell lymphoma cells while promoting the growth of myeloma and waldenstrom macroglobulinemia tumor cells [33-36]. In the present study, we focused on the role of IL-21 in B-ALL cells, to our knowledge which has not been discussed before. As expected, IL-21 promoted the proliferation of Nalm-6 cells and patient B-ALL cells, suggesting that it should work together with IL-17A to facilitate the expansion of B-ALL cells. Furthermore, the addition of IL-21 resulted in robust phosphorylation of Akt and Stat 3 at $15 \mathrm{~min}$ post-stimulation, implying that the PI3K/Akt and Jak2/ Stat3 signaling have been activated quickly, and these two signalings contribute to the proliferation effect.

Daunorubicin as a chemotherapeutic agent has been used clinically to treat B-ALL patients for several decades [37]. Several factors, such as the overexpression of microRNA-125b, influence resistance to daunorubicin [38], and whether IL-17A is an important factor remains unclear. In the present study, IL-17A resulted in resistance to daunorubicin against B-ALL cells, in which the Akt signaling play a key role, because a PI3K/Akt 

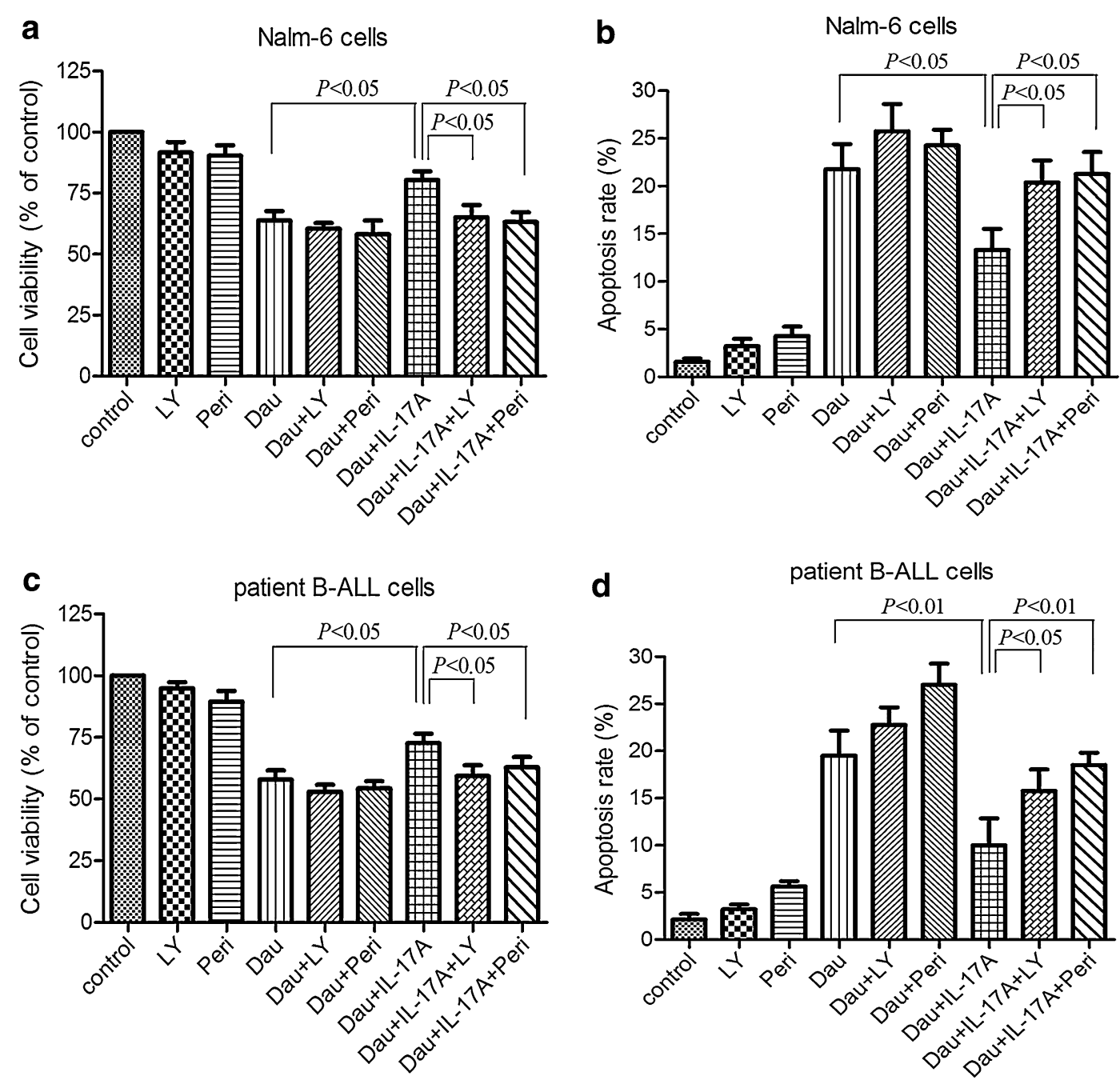

Fig. $5 \mathrm{IL}-17 \mathrm{~A}$-induced resistance to daunorubicin is dependent on Akt activation. Nalm-6 cells $(\mathbf{a}, \mathbf{b})$ and patient B-ALL cells $(\mathbf{c}$, d) isolated from 4 B-ALL patients were pre-treated with the PI3K/Akt inhibitor LY294002 at $10 \mu \mathrm{M}$ or perifosine at $2 \mu \mathrm{M}$ for $2 \mathrm{~h}$ and treated with or without IL-17A at $50 \mathrm{ng} / \mathrm{ml}$ for $24 \mathrm{~h}$. Then, the cells were further treated with daunorubicin at $200 \mathrm{nM}$ for $24 \mathrm{~h}$. The cell viability and apoptosis were determined using CCK-8 assay and Annexin V staining, respectively. Statistical data representing at least three independent experiments were shown

inhibitor LY294002 or perifosine almost blocks the protective effects of IL-17A.

\section{Conclusions}

We found that increased Th17 cells and decreased Th1 cells were presented in $\mathrm{PB}$ and $\mathrm{BM}$ from patients with B-ALL. IL-17A or IL-21 could promote the proliferation of B-ALL cells through activation of Akt and Stat3 signaling. The PI3K/Akt inhibitor LY294002 or perifosine almost completely abrogated IL-17A-mediated protection from daunorubicin-induced cell death in B-ALL cells. Therefore all of the above data suggested that Th17 cells might serve as a potential target for B-ALL immunotherapy.

\section{Additional files}

Additional file 1: Table S1. The sequences of the primers used for realtime $\mathrm{QPCR}$.

Additional file 2: Figure S1. IL-17A reduces apoptosis to daunorubicin in Nalm-6 cells. Nalm-6 cells were cultured for $24 \mathrm{~h}$ in complete medium with or without IL-17A at $50 \mathrm{ng} / \mathrm{ml}$ and subsequently supplemented with daunorubicin at 100, 200, or $400 \mathrm{nM}$ as indicated for $24 \mathrm{~h}$. Apoptosis was determined using Annexin $\vee$ staining on a flow cytometry. Images representing 3 independent experiments were shown.

Additional file 3: Figure S2. IL-17A reduces apoptosis to daunorubicin in patient B-ALL cells. Patient B-ALL cells isolated from 4 patients with B-ALL were cultured for $24 \mathrm{~h}$ in complete medium with or without IL-17A at $50 \mathrm{ng} / \mathrm{ml}$ and subsequently treated with daunorubicin at 100, 200, or $400 \mathrm{nM}$ as indicated for $24 \mathrm{~h}$. Apoptosis was measured using Annexin V staining on a flow cytometry. Images representing at least 3 independent experiments were shown. 


\section{Authors' contributions}

$L B, S Z$ and $Y H$ designed the research study, analyzed the data and wrote the paper; LB, JW, AY, SZ and YH performed the acquisition of data; JW, KY, SZ and $\mathrm{YH}$ analyzed and interpreted the data. All authors read and approved the final manuscript.

\section{Author details}

${ }^{1}$ Department of Hematology, The First Affiliated Hospital of Wenzhou Medical University, Wenzhou 325015, China. ${ }^{2}$ Laboratory of Internal Medicine, the First Affiliated Hospital of Wenzhou Medical University, Wenzhou 325015, China.

\section{Acknowledgements}

This work was supported by National Natural Science Foundation of China (Nos. 81100355, 81172613, 81300430), and Zhejiang Provincial Natural Science Foundation of China (Nos. LQ12H08002, LY16H080007).

\section{Competing interests}

The authors declare that they have no competing interests.

Received: 10 November 2015 Accepted: 4 May 2016

Published online: 12 May 2016

\section{References}

1. Inaba H, Greaves M, Mullighan CG. Acute lymphoblastic leukaemia. Lancet. 2013;381:1943-55.

2. Purizaca J, Meza I, Pelayo R. Early lymphoid development and microenvironmental cues in B-cell acute lymphoblastic leukemia. Arch Med Res. 2012:43:89-101.

3. Murugaiyan G, da Cunha AP, Ajay AK, Joller N, Garo LP, Kumaradevan S, Yosef N, Vaidya VS, Weiner HL. MicroRNA-21 promotes Th17 differentiation and mediates experimental autoimmune encephalomyelitis. J Clin Invest. 2015;125:1069-80.

4. Mease PJ. Inhibition of interleukin-17, interleukin-23 and the TH17 cell pathway in the treatment of psoriatic arthritis and psoriasis. Curr Opin Rheumatol. 2015;27:127-33.

5. Taleb S, Tedgui A, Mallat Z. IL-17 and Th17 cells in atherosclerosis: subtle and contextual roles. Arterioscler Thromb Vasc Biol. 2015:35:258-64.

6. Muranski P, Restifo NP. Essentials of Th17 cell commitment and plasticity. Blood. 2013;121:2402-14.

7. Han Y, Ye A, Bi L, Wu J, Yu K, Zhang S. Th17 cells and interleukin-17 increase with poor prognosis in patients with acute myeloid leukemia. Cancer Sci. 2014;105:933-42.

8. Wilke CM, Kryczek I, Wei S, Zhao E, Wu K, Wang G, Zou W. Th17 cells in cancer: help or hindrance? Carcinogenesis. 2011;32:643-9.

9. Guery L, Dubrot J, Lippens C, Brighouse D, Malinge P, Irla M, Pot C, Reith W, Waldburger JM, Hugues S. Ag-presenting CpG-activated pDCs prime Th17 cells that induce tumor regression. Cancer Res. 2014;74:6430-40.

10. De Simone V, Franze E, Ronchetti G, Colantoni A, Fantini MC, Di Fusco D, Sica GS, Sileri P, MacDonald TT, Pallone F, et al. Th17-type cytokines, IL-6 and TNF-alpha synergistically activate STAT3 and NF-kB to promote colorectal cancer cell growth. Oncogene. 2015;34:3493-503.

11. Tsukamoto H, Senju S, Matsumura K, Swain SL, Nishimura Y. IL-6-mediated environmental conditioning of defective Th1 differentiation dampens antitumour immune responses in old age. Nat Commun. 2015;6:6702

12. Kennedy R, Celis E. Multiple roles for CD4+T cells in anti-tumor immune responses. Immunol Rev. 2008;222:129-44.

13. Liu X, Ren S, Qu X, Ge C, Cheng K, Zhao RC. Mesenchymal stem cells inhibit Th17 cells differentiation via IFN-gamma-mediated SOCS3 activation. Immunol Res. 2015;61:219-29.

14. Lin H, Tong ZH, Xu QQ, Wu XZ, Wang XJ, Jin XG, Ma WL, Cheng X, Zhou Q, Shi HZ. Interplay of Th 1 and Th17 cells in murine models of malignant pleural effusion. Am J Respir Crit Care Med. 2014;189:697-706.

15. Musuraca G, De Matteis S, Napolitano R, Papayannidis C, Guadagnuolo V, Fabbri F, Cangini D, Ceccolini M, Giannini MB, Lucchesi A, et al. IL-17/IL-10 double-producing T cells: new link between infections, immunosuppression and acute myeloid leukemia. J Transl Med. 2015:13:229.

16. Vardiman JW, Thiele J, Arber DA, Brunning RD, Borowitz MJ, Porwit A, Harris NL, Le Beau MM, Hellstrom-Lindberg E, Tefferi A, Bloomfield CD.
The 2008 revision of the world health organization (WHO) classification of myeloid neoplasms and acute leukemia: rationale and important changes. Blood. 2009;114:937-51.

17. Han Y, Ye A, Zhang Y, Cai Z, Wang W, Sun L, Jiang S, Wu J, Yu K, Zhang S. Musashi-2 silencing exerts potent activity against acute myeloid leukemia and enhances chemosensitivity to daunorubicin. PLoS One. 2015;10:e0136484

18. Zhang S, Zhang Y, Zhuang Y, Wang J, Ye J, Wu J, Yu K, Han Y. Matrine induces apoptosis in human acute myeloid leukemia cells via the mitochondrial pathway and Akt inactivation. PLoS One. 2012;7:e46853.

19. Zhang SH, Zhang Y, Shen J, Zhang S, Chen L, Gu J, Mruk JS, Cheng G, Zhu L, Kunapuli SP, Ding Z. Tumor vascular disrupting agent 5,6-dimethylxanthenone-4-acetic acid inhibits platelet activation and thrombosis via inhibition of thromboxane A2 signaling and phosphodiesterase. J Thromb Haemost. 2013;11:1855-66.

20. Prabhala RH, Pelluru D, Fulciniti M, Prabhala HK, Nanjappa P, Song W, Pai C, Amin S, Tai YT, Richardson PG, et al. Elevated IL-17 produced by $\mathrm{TH} 17$ cells promotes myeloma cell growth and inhibits immune function in multiple myeloma. Blood. 2010;115:5385-92.

21. Tian T, Sun Y, Li M, He N, Yuan C, Yu S, Wang M, Ji C, Ma D. Increased Th22 cells as well as Th17 cells in patients with adult T-cell acute lymphoblastic leukemia. Clin Chim Acta. 2013:426:108-13.

22. Wei L, Laurence A, Elias KM, O'Shea JJ. IL-21 is produced by Th17 cells and drives IL-17 production in a STAT3-dependent manner. J Biol Chem. 2007;282:34605-10

23. Yu H, Lee $H$, Herrmann A, Buettner $R$, Jove R. Revisiting STAT3 signalling in cancer: new and unexpected biological functions. Nat Rev Cancer. 2014;14:736-46.

24. Wang L, Man N, Sun XJ, Tan Y, Cao MG, Liu F, Hatlen M, Xu H, Huang G, Mattlin $M$, et al. Regulation of AKT signaling by ld1 controls $t(8 ; 21)$ leukemia initiation and progression. Blood. 2015;126:640-50.

25. Zhou M, Gu L, Findley HW, Jiang R, Woods WG. PTEN reverses MDM2mediated chemotherapy resistance by interacting with $\mathrm{p} 53$ in acute lymphoblastic leukemia cells. Cancer Res. 2003;63:6357-62.

26. Genovese MC, Greenwald M, Cho CS, Berman A, Jin L, Cameron GS, Benichou O, Xie L, Braun D, Berclaz PY, Banerjee S. A phase II randomized study of subcutaneous ixekizumab, an anti-interleukin-17 monoclonal antibody, in rheumatoid arthritis patients who were naive to biologic agents or had an inadequate response to tumor necrosis factor inhibitors. Arthritis Rheumatol. 2014:66:1693-704.

27. Mclnnes IB, Mease PJ, Kirkham B, Kavanaugh A, Ritchlin CT, Rahman P, van der Heijde D, Landewe R, Conaghan PG, Gottlieb AB, et al. Secukinumab, a human anti-interleukin-17A monoclonal antibody, in patients with psoriatic arthritis (FUTURE 2): a randomised, double-blind, placebocontrolled, phase 3 trial. Lancet. 2015;386:1137-46.

28. Miyahara Y, Odunsi K, Chen W, Peng G, Matsuzaki J, Wang RF. Generation and regulation of human CD4+IL-17-producing $T$ cells in ovarian cancer. Proc Natl Acad Sci USA. 2008;105:15505-10.

29. Sfanos KS, Bruno TC, Maris CH, Xu L, Thoburn CJ, DeMarzo AM, Meeker AK Isaacs WB, Drake CG. Phenotypic analysis of prostate-infiltrating lymphocytes reveals TH17 and Treg skewing. Clin Cancer Res. 2008;14:3254-61.

30. Abousamra NK, Salah El-Din M, Helal R. Prognostic value of Th17 cells in acute leukemia. Med Oncol. 2013:30:732.

31. Han Y, Wu J, Bi L, Xiong S, Gao S, Yin L, Jiang L, Chen C, Yu K, Zhang S. Malignant B cells induce the conversion of CD4+CD25- T cells to regulatory T cells in B-cell non-Hodgkin lymphoma. PLoS One. 2011;6:e28649.

32. Ettinger $R$, Sims GP, Fairhurst $A M$, Robbins $R$, da Silva $Y S$, Spolski $R$, Leonard WJ, Lipsky PE. IL-21 induces differentiation of human naive and memory B cells into antibody-secreting plasma cells. J Immunol. 2005;175:7867-79.

33. Gowda A, Roda J, Hussain SR, Ramanunni A, Joshi T, Schmidt S, Zhang X, Lehman A, Jarjoura D, Carson WE, et al. IL-21 mediates apoptosis through up-regulation of the $\mathrm{BH} 3$ family member BIM and enhances both direct and antibody-dependent cellular cytotoxicity in primary chronic lymphocytic leukemia cells in vitro. Blood. 2008;111:4723-30.

34. Sarosiek KA, Malumbres R, Nechushtan H, Gentles AJ, Avisar E, Lossos IS. Novel IL-21 signaling pathway up-regulates c-Myc and induces apoptosis of diffuse large B-cell lymphomas. Blood. 2010;115:570-80.

35. Menoret E, Maiga S, Descamps G, Pellat-Deceunynck C, Fraslon C, Cappellano M, Moreau P, Bataille R, Amiot M. IL-21 stimulates human myeloma cell growth through an autocrine IGF-1 loop. J Immunol. 2008;181:6837-42 
36. Hodge LS, Ziesmer SC, Yang ZZ, Secreto FJ, Gertz MA, Novak AJ, Ansell SM. IL-21 in the bone marrow microenvironment contributes to IgM secretion and proliferation of malignant cells in Waldenstrom macroglobulinemia. Blood. 2012;120:3774-82.

37. Kim DY, Joo YD, Lim SN, Kim SD, Lee JH, Lee JH, Kim DH, Kim K, Jung CW, Kim I, et al. Nilotinib combined with multiagent chemotherapy for newly diagnosed Philadelphia-positive acute lymphoblastic leukemia. Blood. 2015;126:746-56.

38. Zhou L, Bai H, Wang C, Wei D, Qin Y, Xu X. microRNA125b promotes leukemia cell resistance to daunorubicin by inhibiting apoptosis. Mol Med Rep. 2014;9:1909-16.

\section{Submit your next manuscript to BioMed Central and we will help you at every step:}

- We accept pre-submission inquiries

- Our selector tool helps you to find the most relevant journal

- We provide round the clock customer support

- Convenient online submission

- Thorough peer review

- Inclusion in PubMed and all major indexing services

- Maximum visibility for your research

Submit your manuscript at

www.biomedcentral.com/submit 